\title{
Evidence for an isotropic signature in double vibrational collision-induced Raman scattering: A point-polarizable molecule model
}

\author{
M. Chrysos* and I. A. Verzhbitskiy \\ Institut des Sciences et Technologies Moléculaires d'Angers, Moltech Anjou, UMR Centre National de la Recherche Scientifique 6200, \\ Université d'Angers, 2 Boulevard Lavoisier, F-49045 Angers, France
}

(Received 23 December 2009; published 12 April 2010)

\begin{abstract}
The particularly weak isotropic spectrum of the recently reported [Verzhbitskiy et al., Phys. Rev. A 81, 012702 (2010)] nearly depolarized collision-induced Raman scattering band $\mathrm{SF}_{6}\left(v_{1}\right)+\mathrm{N}_{2}\left(v_{1}\right)$ at room temperature was obtained and is presented here. The spectrum was extracted from high-quality measurements of two independent incident-field polarization scattering components. Its zero-order moment was found to be about 200 times smaller than that of its anisotropic counterpart. Agreement, both in spectral shape and in intensity, was found with predictions based on the dipole-induced dipole polarization model once corrected for the very substantial backinduction, dispersion, and dipole-induced quadrupole-induced dipole interaction mechanisms, all of which were considered within the model framework of two point-polarizable molecules. Quantum-mechanical calculations revealed a large contribution from bound and predissociating dimers that amounts to more than one-third of the total isotropic scattering intensity.
\end{abstract}

DOI: 10.1103/PhysRevA.81.042705

PACS number(s): $34.90 .+\mathrm{q}, 33.20 . \mathrm{Fb}, 33.80 .-\mathrm{b}, 36.20 . \mathrm{Ng}$

\section{INTRODUCTION}

Collision-induced Raman scattering (CIS) has been the subject of intensive research for almost three decades [1]. Among the various possible manifestations of CIS, double vibrational Raman scattering (DRS) is an exotic process. Its essentials can be summarized in a few words. Gas molecules in collision scatter the incident photons. While most of the incident energy escapes, part of it is trapped in the gas and converted to kinetic energy of the colliding molecules or invested to simultaneously promote them to excited vibrational states. DRS is nothing but CIS by pairs of molecules, both of which undergo Raman-allowed vibrational transitions. To study it, one has to record and process light-scattering gas spectra, pinpointing those frequencies that correspond to the sum of the two vibrational transitions. DRS events are extremely unlikely to occur in practice because their observation implies that several conditions are met. Not only must the incident photon and the two gas molecules be involved in a triple encounter, but the two molecules must also spontaneously intercept exactly the amount of energy needed to undergo a transition, and the transitions must occur simultaneously. Whenever gas mixtures are involved, DRS transitions can appear at frequency regions that are essentially free from overtone or combination bands of the single molecules. As a result, DRS spectra by mixtures are expected to be more readily discernible than pure gas DRS spectra because the sum of two vibrational modes involving unlike molecules can only coincide with the frequency of an allowed molecular band by accident.

Over the past 15 years, our group has widely studied CIS via a variety of gases and mixtures and has reported spectra that are extremely weak [2]. But DRS pertains to those collisioninduced processes that are notorious for the low intensities they generate, which can often be much lower than the ones in typical collisional spectra. The first observation of DRS in

*michel.chrysos@univ-angers.fr gases was reported a few years ago by Le Duff [3], seven years after the experimental confirmation by Zoppi and his colleagues of DRS in liquids [4]. Le Duff's study, among other findings, revealed that DRS is almost fully depolarized, giving rise solely to anisotropic spectra [3]. Recently, we provided evidence for DRS in the gas mixture $\mathrm{SF}_{6}-\mathrm{N}_{2}$, where we stressed the almost fully depolarized nature of the band and we reported its anisotropic spectrum [5].

Here we report an observation of isotropic DRS in the same mixture. Such a spectrum, whose existence has been predicted theoretically, is expected to be more than one order of magnitude weaker than its anisotropic counterpart, the latter having been shown in the recent past to be sufficiently strong to whet the interest as a potentially discernible nonstandard collision-induced process $[3,5]$. The importance of and reason for detecting isotropic CIS spectra can be outlined in a single sentence. These spectra stand as a forbidding challenge to the spectroscopist, but they also propose themselves as a particularly sensitive predictive tool for intramolecular and intermolecular properties. This is even more true with their extremely weak DRS signature, evidence of the existence of which is provided below. The difficulty with isotropic CIS, aside from the weak signal it produces, is that stray light from allowed polarized components (such as the intense Rayleigh line) can partially or totally mask the spectrum. This can in part explain why isotropic CIS has thus far not received much attention compared to anisotropic CIS. But vibrational CIS spectra are free from the bothersome Rayleigh component, so the aforementioned difficulty does not arise in DRS, even though polarization effects owing to the finite volume of the two molecules (unaccounted for in this article) are also expected to participate. Thus, once carefully measured, isotropic spectra can be useful devices for assessing values of dispersion force constants or molecular polarizabilities, such as for instance the Hamaker $C_{6}$ coefficient of $\mathrm{SF}_{6}-\mathrm{N}_{2}$ or the quadrupole-quadrupole polarizability $C$ of $\mathrm{N}_{2}$ and $\mathrm{SF}_{6}$, which both have a great role in the studied process, as shown below. 


\section{MEASUREMENTS}

Handmade or industrially prepared mixtures of highly purified sulfur hexafluoride and nitrogen gases were used at room temperature $(T=294.5 \mathrm{~K})$ and at moderate working conditions of pressure and density, which for $\mathrm{N}_{2}$ attained values as high as 92 bars but never exceeded 21 bars for $\mathrm{SF}_{6}$. The DRS $\mathrm{SF}_{6}\left(v_{1}\right)+\mathrm{N}_{2}\left(v_{1}\right)$ band was recorded at the sum $v_{a}+v_{b}\left(v_{a}=775 \mathrm{~cm}^{-1}, v_{b}=2330 \mathrm{~cm}^{-1}\right)$ of the two fundamental vibrational stretching frequencies for the two molecules, according to a strict protocol developed in detail in the preceding paper [5]. Two independent scattering signal components, $I_{\|}$and $I_{\perp}$, were measured as a function of Raman frequency shift $v$, depending on whether the electric field of the incident beam was polarized in the direction parallel or perpendicular to the scattering plane [6]. To avoid bias in the polarization of the output electromagnetic field caused by any possible preference of the holographic gratings for one polarization over another, due care was taken to ensure a completely unpolarized signal before the scattered radiation entered the detection chain. A regime strictly quadratic in density was ensured, in which integrated signals scaled as $\rho_{a} \rho_{b}$ products (with $\rho_{a}$ and $\rho_{b}$ as the densities of the two gases) for both the parallel and the perpendicular polarization configurations. This property offered an unambiguous guarantee of binary $\mathrm{SF}_{6}-\mathrm{N}_{2}$ scattering. The DRS band was found to be nearly fully depolarized, with a near constant depolarization ratio $\eta(v)=0.83 \pm 0.02$ over the rather narrow frequency domain $3080-3120 \mathrm{~cm}^{-1}$, out of which extraction of an isotropic signal was possible. This property proved to be particularly useful for the meaningful determination of the weak isotropic spectrum. To this end, the least-squares fitting method was used to fit the function $I_{0} \exp \left(A_{4} v^{4}+\cdots+A_{1} v\right)$ to the two sets of recorded data for the calibrated Stokes intensities $I_{\perp}$ and $I_{\|}$, with parameter values for $A_{1}, \ldots, A_{4}$ common to both spectral components (thereby taking account of the fact that the ratio $\eta=I_{\|} / I_{\perp}$ was constant over the aforementioned frequency range), but with a parameter value $I_{0}$ that was different in each of them.

Figure 1 illustrates, in absolute units $\left(\mathrm{cm}^{6}\right)$, the recorded components $I_{\perp}$ and $I_{\|}$in the Stokes side of the spectrum as a function of frequency shift (in $\mathrm{cm}^{-1}$ ) with respect to the center of the band. ${ }^{1}$

The two sets of converged parameters $I_{0}, A_{1}, \ldots, A_{4}$ ensured an excellent fit to both $I_{\perp}$ and $I_{\|}$data and so, too, was the case with the anisotropic and isotropic spectra, $I_{\text {ani }}$ and $I_{\text {iso }}$, which are given as the linear combinations

$$
\begin{gathered}
I_{\mathrm{ani}}=1.01 I_{\|}-0.01009 I_{\perp}, \\
I_{\mathrm{iso}}=1.017 I_{\perp}-1.184 I_{\|} .
\end{gathered}
$$

\footnotetext{
${ }^{1}$ We take this opportunity to point out an inadvertent error in our previous related work [5]. Figure 4, therein, underrates by a factor of 1.11 and 1.15 the signals $I_{\|}$and $I_{\perp}$, respectively. This error escaped notice in Fig. 4 even though it was corrected elsewhere before publication [5]. This graphical error has now been rectified in Fig. 1 of the present article, where $I_{\|}$and $I_{\perp}$ are shown in the same scale as in our preceding article.
}

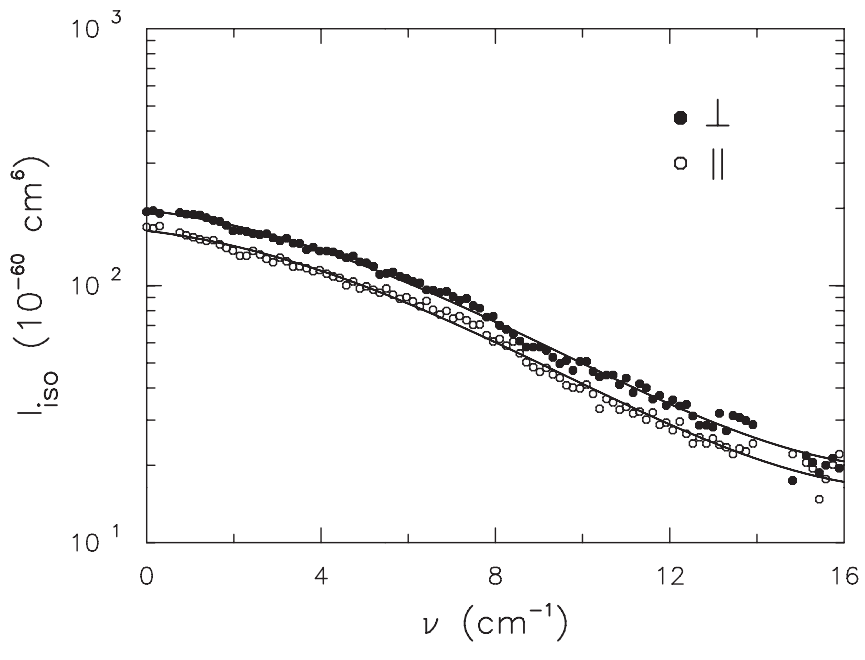

FIG. 1. Absolute components $I_{\perp}(\bullet)$ and $I_{\|}(\circ)$ recorded in the Stokes side of the spectrum, in units of $\mathrm{cm}^{6}$, as a function of frequency shift (in $\mathrm{cm}^{-1}$ ) with respect to the center of the band. Semilogarithmic scale is used. The function $I_{0} \exp \left(A_{4} v^{4}+\cdots+A_{1} v\right)$ for either of the two components is represented by solid lines.

The coefficients in these two expressions account properly for the finite aperture of the scattered beam, as a replacement of the traditional expressions $I_{\text {ani }}=I_{\|}$and $I_{\text {iso }}=I_{\perp}-\frac{7}{6} I_{\|}$, which are valid only in the strictly ideal case of a zero aperture. In this way a high-quality analytic function was obtained for $I_{\text {ani }}$, which reads

$$
I_{\text {ani }}=I_{0} \exp \left(A_{1} v+A_{2} v^{2}+A_{3} v^{3}+A_{4} v^{4}\right)
$$

with parameters $A_{1}=-0.04639, A_{2}=-0.01116, A_{3}=$ $3.66674 \times 10^{-6}$, and $A_{4}=2.03481 \times 10^{-5}$ and with amplitude $I_{0}=1.6329 \times 10^{-58}$. For the isotropic spectrum, $I_{\text {iso }}$, the best value for the amplitude is $I_{0}^{\prime}=6.1301 \times 10^{-60}$. This value corresponds to a depolarization ratio of 0.83 , which was nevertheless subject to variations within the interval $0.81-0.85$. This interval was established by taking into account any error in the measured signals $I_{\|}$and $I_{\perp}$. The small dispersion of the data points ( $\mathrm{O}$ and $\bullet$ ) around the plotted curves of Fig. 1 bears witness to the quality of the data for those two signals.

The variations in $\eta$, albeit small, had a huge effect on the isotropic spectrum, whose integrated intensity suffered variations by more than a factor of 5 . The mean value of the zero-order isotropic moment was found to be $M_{0}=$ $2.92 \times 10^{-4} a_{0}^{9}$ (where $a_{0}$ is the Bohr radius) with lowest and highest acceptable bounds $0.88 \times 10^{-4}$ and $5.07 \times 10^{-4} a_{0}^{9}$, respectively. The latter values determine an area, shown in gray in Fig. 2, which according to our measurements should delimit the uncertainty of the physical isotropic spectrum. The lower bound corresponds to the upper limit of the depolarization ratio (i.e., 0.85), whereas the upper bound refers to the lower limit of 0.81. In Fig. 2, the mean isotropic spectrum is indicated by a dashed line. To assess the relative magnitude of the experimental uncertainty in extracting the isotropic spectrum, the dispersion of the symbols used to depict the data points in Fig. 2 (o) is again a good device. For comparison, the data points for the anisotropic spectrum are also illustrated $(\bullet)$, along with the corresponding $I_{0} \exp \left(A_{1} v+\cdots+A_{4} v^{4}\right)$ 


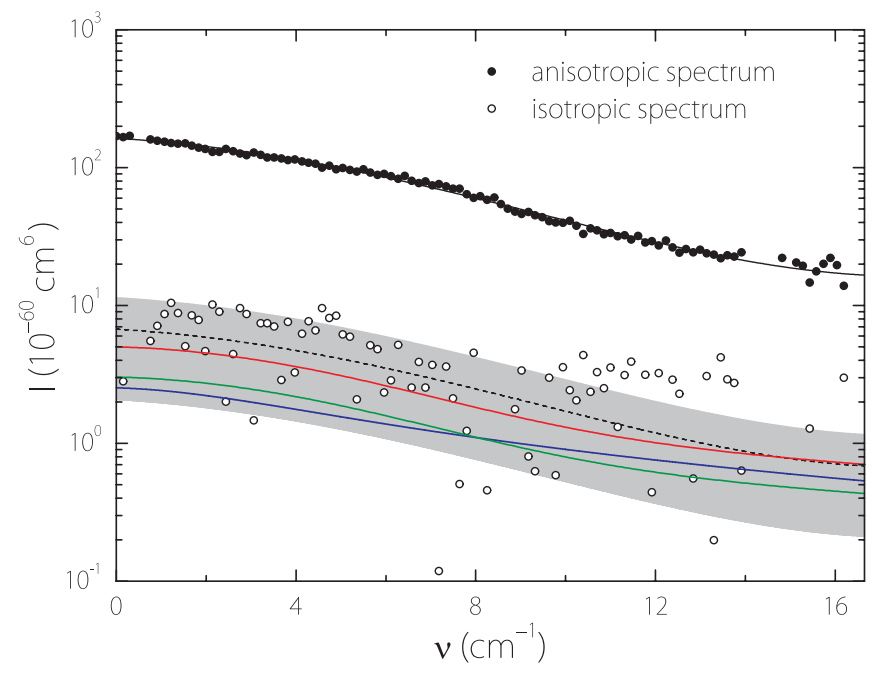

FIG. 2. (Color online) Isotropic spectrum (o) extracted from the recorded absolute components $I_{\perp}$ and $I_{\|}$(in $\mathrm{cm}^{6}$ ) as a function of frequency shift (in $\mathrm{cm}^{-1}$ ) with respect to the center of the band. Semilogarithmic scale is used. The area marked in gray defines the uncertainty boundaries of the physical isotropic spectrum. The mean spectrum is indicated by a dashed line. For comparison, data points for the anisotropic spectrum are also shown $(\bullet)$, along with mean intensities (thin solid line). Thick solid lines are used to depict quantum-mechanical isotropic spectra, computed within the point-polarizable molecule interaction model and with inclusion of DID polarization terms through orders $R^{-6}$ and $R^{-9}$, dispersion polarization through order $R^{-6}$, and $\mathrm{DIQ}^{(2)}$ polarization through order $R^{-8}$, for different $C_{6}$ entry values and types of function for pressure effects. These are (from bottom to top at $v=0$ ): $C_{6}=247$ a.u., Lorentzian with a full width at half-maximum equal to $10 \mathrm{~cm}^{-1}$ [blue (bottom) line]; $C_{6}=247$ a.u., Gaussian with a full width at half-maximum equal to $10 \mathrm{~cm}^{-1}$ [green (middle) line ]; and $C_{6}=$ 768 a.u., Gaussian with a full width at half-maximum equal to $10 \mathrm{~cm}^{-1}$ [red (upper) line].

function (thin solid line). Particularly gratifying was the fact that theory, within the point-polarizable molecule interaction model and upon inclusion of dipole-induced dipole (DID) polarization and back-induction, through orders $R^{-6}$ and $R^{-9}$, as well as dispersion polarization (through order $R^{-6}$ ) and dipole-induced quadrupole-induced dipole interaction $\left(\mathrm{DIQ}^{(2)}\right.$ ) contributions (through order $R^{-8}$ ), predicts, on the basis of quantum mechanics, a spectrum that lies in the deep interior of the gray zone of Fig. 2. This spectrum is illustrated by three thick solid lines, depending on the choice of the entry value for Hamaker constant $C_{6}$ and on whether a Lorentzian or a Gaussian function was taken to semiempirically simulate part of the effects of the gas pressure (see below). Such an agreement shown in Fig. 2 between theory and experiment lends credence to both the employed polarization model and the adopted procedure for the extraction of particularly weak spectra in nearly depolarized systems. According to our calculations, the effect of dispersion polarization on the isotropic intensity is about one-third that of the total effect of the $R^{-6}$-order polarizability terms; $\mathrm{DIQ}^{(2)}$ is responsible for more than $40 \%$ of the total translational isotropic spectrum. A detailed description of the calculations of the incremental polarizability trace and of the isotropic spectrum and its moment is made in the next section.

\section{CAlCulations}

\section{A. Trace and moments}

Unlike the case of the recently reported anisotropic spectrum [5], for which DID was the sole mechanism responsible for the leading order, here dispersion terms need to be considered from the outset as these terms now participate along with DID terms through the leading order. To this purpose, a rigorous derivation of DID and dispersion effects through order $R^{-6}$, supplemented with $\mathrm{DIQ}^{(2)}$ and back-induction corrections through orders $R^{-8}$ and $R^{-9}$, is given below. Mean and first stretching-derivative values for dipole polarizability $\alpha_{c}(c=a, b)$, quadrupole polarizability $C_{c}$, and second dipole hyperpolarizability $\gamma_{c}$ were necessary as well as structural properties for $a$ and $b$. Henceforth, notations $a$ and $b$ are used to denote molecules $\mathrm{SF}_{6}$ and $\mathrm{N}_{2}$, respectively. To calculate DID, back-induction, and dispersion polarization effects, use is made hereafter of an appendix and of mathematical steps that go beyond the formulas reported in [7].

The incremental trace of the $\mathrm{SF}_{6}-\mathrm{N}_{2}$ polarizability reads $\Delta \alpha=\frac{1}{3}\left(\alpha_{Z Z}+2 \alpha_{X X}\right)$. According to [7], there are only three terms $(0 \Lambda L)$ contributing to $\Delta \alpha$, namely the terms $(000)$, (022), and (044); $\Lambda$ and $L$ designate angular momentum quantum numbers that enter spherical harmonics $Y_{\Lambda}^{m}\left(\Omega_{b}\right)$ and $Y_{L}^{-m}(\Omega)$, where $\Omega_{b}$ specifies the orientation of $b$, and $\Omega$ the orientation of the pair. While the term (044) scales as $R^{-5}$, as compared to the shorter-range term (000), the property $\Delta \alpha_{044}$ introduces components $E_{X, X X X}$ and $E_{Z, Z Z Z}$ of the dipole-octopole polarizability tensor, which vanishes for spherically symmetric systems. The first of the remaining two terms reads [7]

$$
\begin{aligned}
\Delta \alpha_{000}= & 2 \alpha_{a} \alpha_{b}\left(\alpha_{a}+\alpha_{b}\right) R^{-6} \\
& +\frac{\hbar}{\pi} \int_{0}^{\infty} d \omega\left\{\alpha_{a}(i \omega)\left[3 \gamma_{1 b}(i \omega, 0,0)+2 \gamma_{2 b}(i \omega, 0,0)\right]\right. \\
& \left.+\left[3 \gamma_{1 a}(i \omega, 0,0)+2 \gamma_{2 a}(i \omega, 0,0)\right] \alpha_{b}(i \omega)\right\} R^{-6}
\end{aligned}
$$

The second term, $\Delta \alpha_{022}$, involves the anisotropy of $b$, which in the point-polarizable molecule model is set equal to zero, as well as quantities $\gamma_{3 b}$ and $\gamma_{5 b}$ [7], which vanish too. Specifically, in the model adopted here, the $\hat{\gamma}$-tensor elements are reduced to $\gamma_{Z Z X X}=\gamma_{X Z X Z}=\gamma_{X X Z Z}=\gamma_{X X Y Y}=\frac{1}{3} \gamma_{Z Z Z Z}=$ $\frac{1}{3} \gamma_{X X X X}$ for either particle. The four quantities $\gamma_{1 c}, \gamma_{2 c}, \gamma_{3 c}$, and $\gamma_{5 c}$, shown above for particle $c(=a, b)$, are then reduced to $\frac{1}{3} \gamma_{Z Z Z Z}, \frac{1}{3} \gamma_{Z Z Z Z}, 0$, and 0 , respectively. As a result, $\Delta \alpha_{000}$ is the sole nonzero element, which then reads

$$
\begin{aligned}
\Delta \alpha_{000}= & 2 \alpha_{a} \alpha_{b}\left(\alpha_{a}+\alpha_{b}\right) R^{-6} \\
& +\frac{5 \hbar}{3 \pi} \int_{0}^{\infty} d \omega\left[\alpha_{a}(i \omega) \gamma_{b}(i \omega, 0,0)\right. \\
& \left.+\gamma_{a}(i \omega, 0,0) \alpha_{b}(i \omega)\right] R^{-6},
\end{aligned}
$$

where $\gamma_{a}\left(\gamma_{b}\right)$ now denotes the sole independent nonzero element $\gamma_{Z Z Z Z}$ for particle $a(b)$ [8]. 
Equation (4) can be written as

$$
\begin{aligned}
\Delta \alpha_{000}= & 2 \alpha_{a} \alpha_{b}\left(\alpha_{a}+\alpha_{b}\right) R^{-6} \\
& +\frac{5}{9} C_{6}\left[\frac{\int_{0}^{\infty} \alpha_{a}(i \omega) \gamma_{b}(i \omega, 0,0) d \omega}{\int_{0}^{\infty} \alpha_{a}(i \omega) \alpha_{b}(i \omega) d \omega}\right. \\
& \left.+\frac{\int_{0}^{\infty} \gamma_{a}(i \omega, 0,0) \alpha_{b}(i \omega) d \omega}{\int_{0}^{\infty} \alpha_{a}(i \omega) \alpha_{b}(i \omega) d \omega}\right] R^{-6},
\end{aligned}
$$

where

$$
C_{6}=\frac{3 \hbar}{\pi} \int_{0}^{\infty} \alpha_{a}(i \omega) \alpha_{b}(i \omega) d \omega .
$$

By applying Unsöld's approximation [9], the property $\Delta \alpha_{000}$ is further reduced to

$$
\Delta \alpha_{000} \approx 2 \alpha_{a} \alpha_{b}\left(\alpha_{a}+\alpha_{b}\right) R^{-6}+\frac{5}{18} C_{6}\left(\frac{\gamma_{a}}{\alpha_{a}}+\frac{\gamma_{b}}{\alpha_{b}}\right) R^{-6},
$$

where $\alpha_{a}$ and $\gamma_{a}\left(\alpha_{b}\right.$ and $\left.\gamma_{b}\right)$ now denote static properties for particle $a(b)$. Note that the latter expression coincides with the one suggested (though without demonstration) by Hohm for two unlike interacting atoms [10].

In the present case, however, matrix elements $\left\langle 1_{a} 1_{b}\left|\Delta \alpha_{000}\right| 0_{a} 0_{b}\right\rangle$ between vibrational states are to be considered rather than the mean value of $\Delta \alpha_{000}$. As a result, Eq. (7) must further be processed in order to clear away denominators and to account properly for the coupling between the vibrational degrees of freedom in the two molecules on which $C_{6}$ depends intrinsically. Equation (7) is thus replaced by the expression

$$
\Delta \alpha_{000} \approx 2 \alpha_{a} \alpha_{b}\left(\alpha_{a}+\alpha_{b}\right) R^{-6}+\frac{5}{12} \hbar \bar{\omega}\left(\alpha_{a} \gamma_{b}+\gamma_{a} \alpha_{b}\right) R^{-6} .
$$

The latter expression is valid under the well-known London approximation [11]

$$
\int_{0}^{\infty} \alpha_{a}(i \omega) \alpha_{b}(i \omega) d \omega \approx \frac{\pi}{2} \bar{\omega} \alpha_{a} \alpha_{b}
$$

where $\bar{\omega}$ denotes the reduced frequency $\frac{\bar{\omega}_{a} \bar{\omega}_{b}}{\bar{\omega}_{a}+\bar{\omega}_{b}}$ and $\bar{\omega}_{a}$ $\left(\bar{\omega}_{b}\right)$ is some effective characteristic frequency for molecule $a(b)$ [12].

Upon application of the kets and bras, which are associated with the vibrational transitions in the two particles, the following matrix element is obtained:

$$
\left\langle 1_{a} 1_{b}\left|\Delta \alpha_{000}\right| 0_{a} 0_{b}\right\rangle=\mathcal{A} R^{-6}
$$

with

$$
\begin{aligned}
\mathcal{A} \approx & \frac{\hbar}{2}\left(\mu_{a} \mu_{b} \omega_{a} \omega_{b}\right)^{-1 / 2}\left\{4\left(\alpha_{a}^{\prime}\right)_{r_{e}^{a}}\left(\alpha_{b}^{\prime}\right)_{r_{e}^{b}}\left[\left(\alpha_{a}\right)_{r_{e}^{a}}+\left(\alpha_{b}\right)_{r_{e}^{b}}\right]\right. \\
& \left.+\frac{5}{12} \hbar \bar{\omega}\left[\left(\alpha_{a}^{\prime}\right)_{r_{e}^{a}}\left(\gamma_{b}^{\prime}\right)_{r_{e}^{b}}+\left(\gamma_{a}^{\prime}\right)_{r_{e}^{a}}\left(\alpha_{b}^{\prime}\right)_{r_{e}^{b}}\right]\right\} .
\end{aligned}
$$

The next correction scales as $R^{-8}$. It arises because the gradient of the field of the dipole induced in molecule $a(b)$ by the external field acts through the quadrupole polarizability $C_{a}\left(C_{b}\right)$ to induce a quadrupole in molecule $b(a)$ whose field in turn contributes to the induction of a dipole in $a(b)$. This mechanism is often referred to as the dipole-induced quadrupole-induced dipole interaction, or, in an abbreviated terminology, the second-order dipole-induced quadrupole interaction $\left(\mathrm{DIQ}^{(2)}\right)$ [13]. Although this is a short-range term as compared to the leading-order ones, its contribution to isotropic DRS was found to be great, owing principally to interferences with the leading-order mechanisms. In light of our findings, we expect this term to play a substantial role in general in the polarized component of induced spectra, whether the process is DRS or not. The importance of this term to the polarized component of CIS has already been stressed in prior studies with inert gas atoms [13].

Correction to account for $\mathrm{DIQ}^{(2)}$ effects gives $\left\langle 1_{a} 1_{b}\left|10\left(\alpha_{a}^{2} C_{b}+C_{a} \alpha_{b}^{2}\right)\right| 0_{a} 0_{b}\right\rangle R^{-8}=\mathcal{B} R^{-8}$, where

$$
\begin{aligned}
\mathcal{B} \approx & 10 \hbar\left(\mu_{a} \mu_{b} \omega_{a} \omega_{b}\right)^{-1 / 2}\left[\left(\alpha_{a}\right)_{r_{e}^{a}}\left(\alpha_{a}^{\prime}\right)_{r_{e}^{a}}\left(C_{b}^{\prime}\right)_{r_{e}^{b}}\right. \\
& \left.+\left(C_{a}^{\prime}\right)_{r_{e}^{a}}\left(\alpha_{b}\right)_{r_{e}^{b}}\left(\alpha_{b}^{\prime}\right)_{r_{e}^{b}}\right] .
\end{aligned}
$$

Note that for two identical molecules the DIQ ${ }^{(2)}$ term reduces to the familiar $20 \alpha_{0}^{2} C R^{-8}$ expression.

Back-induction through order $R^{-9}$ gives [see Eq. (A8) of the appendix] the supplementary term $\left\langle 1_{a} 1_{b}\left|4 \alpha_{a}^{2} \alpha_{b}^{2}\right| 0_{a} 0_{b}\right\rangle R^{-9}=\mathcal{C} R^{-9}$, where

$$
\mathcal{C} \approx 8 \hbar\left(\mu_{a} \mu_{b} \omega_{a} \omega_{b}\right)^{-1 / 2}\left(\alpha_{a}\right)_{r_{e}^{a}}\left(\alpha_{b}\right)_{r_{e}^{b}}\left(\alpha_{a}^{\prime}\right)_{r_{e}^{a}}\left(\alpha_{b}^{\prime}\right)_{r_{e}^{b}} .
$$

To obtain the latter three expressions, quantities $\alpha_{a}, \gamma_{a}$, and $C_{a}$ $\left(\alpha_{b}, \gamma_{b}\right.$, and $\left.C_{b}\right)$ had to be expanded in a Taylor series about the equilibrium position as a function of the stretching coordinate $q_{1}^{a}\left(q_{1}^{b}\right)$ for molecule $a(b)$ and then truncated to the linear term according to the expression

$$
X_{a} \approx\left(X_{a}\right)_{e}+\left(\frac{\partial X_{a}}{\partial q_{1}^{a}}\right)_{e} q_{1}^{a},
$$

where $X_{a}$ stands for $\alpha_{a}, \gamma_{a}$, and $C_{a}$; analogous expressions apply to molecule $b$. Equations (11)-(13) were then obtained by taking the matrix element of the resulting terms between ground and excited vibrational states for the two particles.

The value of $\bar{\omega}$ for $\mathrm{SF}_{6}-\mathrm{N}_{2}$ is an unknown quantity but its appearance in the final formulas can be avoided by again using Eq. (9) along with the definition of $C_{6}$ [Eq. (6)]: $\hbar \bar{\omega} \approx$ $2 C_{6} /\left(3 \alpha_{a} \alpha_{b}\right) \approx 2 C_{6} /\left[3\left(\alpha_{a}\right)_{r_{e}^{a}}\left(\alpha_{b}\right)_{r_{e}^{b}}\right]$. Note that, in the latter expression, the product $\alpha_{a} \alpha_{b}$ was simplified by the product of the mean polarizabilities, $\left(\alpha_{a}\right)_{r_{e}^{a}}\left(\alpha_{b}\right)_{r_{e}^{b}}$. Equation (11) then reads

$$
\begin{aligned}
\mathcal{A} \approx & \frac{\hbar}{2}\left(\mu_{a} \mu_{b} \omega_{a} \omega_{b}\right)^{-1 / 2}\left\{4\left(\alpha_{a}^{\prime}\right)_{r_{e}^{a}}\left(\alpha_{b}^{\prime}\right)_{r_{e}^{b}}\left[\left(\alpha_{a}\right)_{r_{e}^{a}}+\left(\alpha_{b}\right)_{r_{e}^{b}}\right]\right. \\
& \left.+\frac{5}{18} C_{6} \frac{\left(\alpha_{a}^{\prime}\right)_{r_{e}^{a}}\left(\gamma_{b}^{\prime}\right)_{r_{e}^{b}}+\left(\gamma_{a}^{\prime}\right)_{r_{e}^{a}}\left(\alpha_{b}^{\prime}\right)_{r_{e}^{b}}}{\left(\alpha_{a}\right)_{r_{e}^{a}}\left(\alpha_{b}\right)_{r_{e}^{b}}}\right\} .
\end{aligned}
$$

Interestingly enough, the vibrational coupling between particles $a$ and $b$ has now been shifted, as suggested by Eq. (15), to the products $\left(\alpha_{a}^{\prime}\right)_{r_{e}^{a}}\left(\gamma_{b}^{\prime}\right)_{r_{e}^{b}}$ and $\left(\gamma_{a}^{\prime}\right)_{r_{e}^{a}}\left(\alpha_{b}^{\prime}\right)_{r_{e}^{b}}$, whereas initially the coupling between the two particles was ensured through Eq. (6). The constant $C_{6}$ in Eq. (15) has now become a static property. For our calculations, the experimental value $C_{6}=247 E_{\mathrm{h}} a_{0}^{6}$ [14] was used. For comparison, the far greater Lennard-Jones value $C_{6}=768 E_{\mathrm{h}} a_{0}^{6}$ was also taken (see Ref. [17] in our preceding paper [5]) to calculate frequencyresolved spectra, which compares even more favorably with our measurements. 
TABLE I. Input parameters for molecule $c(=a, b)$ and for the pair $a$ - $b$. All values are in atomic units (a.u.). The best values, used as input for our calculations, are denoted by bold entries. The entry for $\left(\alpha_{a}\right)_{r_{e}^{a}}$ is very close to the experimental value of 30.35 a.u. reported in [15].

\begin{tabular}{lcccccc}
\hline \hline$c$ & $\left(\alpha_{c}\right)_{r_{e}^{c}}$ & $\left(\alpha_{c}^{\prime}\right)_{r_{e}^{c}}$ & $\left(\gamma_{c}^{\prime}\right)_{r_{e}^{c}}$ & $\left(C_{c}^{\prime}\right)_{r_{e}^{c}}$ & $\sqrt{\frac{\hbar}{2 \mu_{c} \omega_{c}}}$ & $C_{6}$ \\
\hline$a$ & $26.54^{\mathrm{a}}$ & & $813^{\mathrm{a}}$ & & 0.0261 & \\
& $\mathbf{3 0 . 2 9}^{\mathrm{b}}$ & $25.38^{\mathrm{b}}$ & $\mathbf{9 1 5}^{\mathrm{b}}$ & $257.8^{\mathrm{b}}$ & & \\
& & $\mathbf{2 6 . 3 5}^{\mathrm{c}}$ & & & & \\
& & & $642^{\mathrm{d}}$ & & & \\
$b$ & $11.85^{\mathrm{f}}$ & $6.176^{\mathrm{f}}$ & $994^{\mathrm{f}}$ & $23.31^{\mathrm{f}}$ & 0.0607 & \\
$a-b$ & & & & & & $\mathbf{2 4 7}^{\mathrm{g}}$ \\
& & & & & & $205.8^{\mathrm{h}}$ \\
& & & & & & $768^{\mathrm{i}}$ \\
\hline \hline
\end{tabular}

a SCF values for molecule $a$ [16].

${ }^{\mathrm{b}} \mathrm{MP} 2$ values for molecule $a$ [16].

${ }^{\mathrm{c} E x p e r i m e n t a l}$ value from [18].

dMaroulis's SCF value [16] but with use of the invariant reported in [7] for $\mathrm{SF}_{6}$ (see [8]).

eMaroulis's MP2 value [16] but with use of the invariant reported in [7] for $\mathrm{SF}_{6}$.

${ }^{\mathrm{f}} \mathrm{CCSD}(\mathrm{T})$ values for molecule $b$ [17].

${ }^{\mathrm{g}}$ Experimental value from [14].

${ }^{\text {h }}$ Most recent theoretical study [19], which is $20 \%$ below the measured $C_{6}$ value of $\mathrm{SF}_{6}-\mathrm{N}_{2}$.

${ }^{\mathrm{i}} C_{6}$ value for a Lennard-Jones potential with $\varepsilon=138.8 \mathrm{~K}$ and $\sigma=$ $8.71 a_{0}$.

Input parameters relative to molecules $a$ and $b$ and to the pair $a-b$ are given in Table I. For the mean static polarizability of $\mathrm{SF}_{6}$, Maroulis's second-order Møller-Plesset (MP2) value was used [16] (bold entry), which is very close to the experimental value 30.35 a.u. reported elsewhere [15]. For $\mathrm{N}_{2}$, Maroulis's CCSD(T) (coupled cluster with single, double, and partially triple excitations) values were used for both the mean and the first derivative of the dipole polarizability as well as for the first derivatives of the second dipole hyperpolarizability and of the quadrupole polarizability of this molecule [17]. While the expression for the isotropic invariant of the second dipole hyperpolarizability of $\mathrm{N}_{2}$ given by Maroulis agrees with that reported in [7], in the case of $\mathrm{SF}_{6}$ the two authors have reported two different expressions; however, both expressions lead to the same formal result when the molecules are treated as point-polarizable particles, which is the model used throughout this paper (see [8]). For the first polarizability derivative of $\mathrm{SF}_{6}$, the experimental value was taken [18], even though ab initio results for self-consistent field (SCF) or MP2 level are also available [16]. For the quadrupole polarizability derivative, the MP2 value of [16] was used. For the Hamaker constant $C_{6}$ that enters dispersion terms, the experimental value was used [14]. Although more recent $C_{6}$ values do exist, they are theoretically obtained [19]. Note that the experimental value used in our calculations is $20 \%$ above the most recent theoretical value. The highest value of $C_{6}$ was the one associated with the Lennard-Jones potential model (Ref. [17] in [5]). Values for the reduced masses $\mu_{a}$ and $\mu_{b}$ are 114 and 7 amu, respectively. Angular frequency $\omega_{c}$ is defined
TABLE II. Values (in a.u.) of coefficients $\mathcal{A}$ [Eq. (15)], $\mathcal{B}$ [Eq. (12)], and $\mathcal{C}$ [Eq. (13)]. For $\mathcal{A}$, the case of DID alone and that of DID supplemented with dispersion effects were separately considered.

\begin{tabular}{lccc}
\hline \hline $\mathcal{A}(\mathrm{DID})$ & $\mathcal{A}(\mathrm{DID}+\mathrm{disp})$ & $\mathcal{B}\left(\mathrm{DIQ}^{(2)}\right)$ & $\mathcal{C}(\mathrm{DID})$ \\
\hline 43.5 & 53.1 & 1188 & 1482 \\
\hline \hline
\end{tabular}

as $\omega_{c}=2 \pi c v_{c}$, with $v_{a}=775 \mathrm{~cm}^{-1}\left(v_{b}=2330 \mathrm{~cm}^{-1}\right)$ as the characteristic wavenumber of the vibrational transition in molecule $a(b)$. Finally, as the $\mathrm{SF}_{6}-\mathrm{N}_{2}$ potential, the most updated interaction energy model was used throughout [20], which has been clearly proven to be superior to any other prior model for $\mathrm{SF}_{6}-\mathrm{N}_{2}$ [5], even if still insufficient for accurate quantitative predictions.

The values for $\mathcal{A}, \mathcal{B}$, and $\mathcal{C}$ are given in Table II, with and without inclusion of the dispersion polarization mechanism.

When passing to moments, $M_{0}$ was the only quantity to provide a converged value in the restricted range that was covered experimentally. This quantity was obtained by using the general expression

$$
M_{0}=4 \pi \int_{0}^{\infty}(\Delta \alpha)^{2} G(R) R^{2} d R
$$

where $G(R)$ is the binary distribution function $e^{-V(R) / k_{B} T}$, and $V(R)$ and $k_{B}$ are the isotropic effective interaction potential and the Boltzmann constant, respectively. After some simple algebra, we obtain

$$
\begin{aligned}
M_{0}= & \mathcal{A}^{2} \mathcal{I}_{10}+2 \mathcal{A B \mathcal { I } _ { 1 2 }}+2 \mathcal{A C \mathcal { I } _ { 1 3 }}+\mathcal{B}^{2} \mathcal{I}_{14} \\
& +2 \mathcal{B C} \mathcal{I}_{15}+\mathcal{C}^{2} \mathcal{I}_{16},
\end{aligned}
$$

where $\mathcal{I}_{p}(p=10,12, \ldots, 16)$ is the integral

$$
\mathcal{I}_{p}=4 \pi \int_{0}^{\infty} e^{-V(R) / k_{B} T} R^{-p} d R .
$$

Values of $M_{0}$, which according to Eqs. (12), (13), and (15) account for all or part of the contributions to the integrated intensity, were calculated by means of Eq. (17). The six partial contributions of this equation amount (in the order of their appearance in the equation) to $0.953 \times 10^{-4}, 0.620 \times 10^{-4}$, $0.941 \times 10^{-5}, 0.103 \times 10^{-4}, 0.317 \times 10^{-5}$, and $0.244 \times 10^{-6}$ $a_{0}^{9}$. The values were compared to the moment obtained experimentally through the expression

$$
M_{0}^{\operatorname{expt}}=\left(\frac{\lambda}{2 \pi}\right)^{4} \int_{-\infty}^{\infty} I_{\mathrm{iso}}(\nu) d \nu,
$$

where $\lambda=\left(v_{L}-v_{a+b}\right)^{-1}$, and $v_{L}$ and $v_{a+b}$ denote the wavenumbers of the laser beam and of the DRS signal at the peak intensity, respectively.

Table III gathers values for $M_{0}$ in $10^{-4} a_{0}^{9}$ units. The second column shows the value due to DID plus dispersion through the leading order. The third column gives the $M_{0}$ value after inclusion of $\mathrm{DIQ}^{(2)}$ corrections. The fourth column shows the converged moment value after second-order DID corrections were added. Interestingly enough, the corrections are responsible for an increase in the integrated intensity by a 
TABLE III. Values for the zero-order isotropic moment $M_{0}$ in $10^{-4} a_{0}^{9}$ units. The first four columns refer to theory (this work). "expt." gives the range of the tolerated moment values obtained experimentally (this work). The mean (best) experimental value is indicated in parentheses.

\begin{tabular}{lcccc}
\hline \hline $\mathrm{DID}\left(R^{-6}\right)$ & $+\operatorname{disp}\left(R^{-6}\right)$ & $+\mathrm{DIQ}^{(2)}\left(R^{-8}\right)$ & $+\mathrm{DID}\left(R^{-9}\right)$ & expt. \\
\hline 0.64 & 0.95 & 1.68 & 1.80 & $\begin{array}{c}0.9-5.7 \\
\end{array}$ \\
& & & & $(2.9)$ \\
\hline \hline
\end{tabular}

factor of 3 with most of this increase coming from dispersion and $\mathrm{DIQ}^{(2)}$ terms.

\section{B. The spectrum}

Spectra were calculated quantum-mechanically by means of the Fermi golden rule for the scattering cross sections. The wave functions of the supermolecule were built step by step on a 7600-point grid nearly 300 bohrs long, by means of the propagative Fox-Goodwin method previously developed by one of us [21]. Matrix elements between states of the continuum were computed and their convergence was ensured to within $1 \%$. Rotational levels as high as $J=300$ were populated in order to ensure that cross sections were properly converged over the running angular momentum quantum number. Owing to internal symmetry considerations, only $\Delta J=0$ transitions were allowed for isotropic spectra, providing line shapes reputed to decrease exponentially in the wing. As a consequence of the absence of geometric symmetry in $\mathrm{SF}_{6}-\mathrm{N}_{2}$, even and odd values of $J$ contributed on an equal footing to the calculation. A total number of 300 equally spaced energy values were considered in steps of $15 \mathrm{~cm}^{-1}$, allowing us to span the energy domain above the dissociation threshold up to $4500 \mathrm{~cm}^{-1}$ in the deep continuum.

Bound states as well as shape resonances were computed and their spectra were superposed to the unstructured continuum. To this end, an efficient method was used, which was developed by one of us and reported in a previous paper [22]. This method makes use of a discrete variable representation (DVR) and allows one to generate, for each value of $J$, all vibrational bound-state and predissociating-state energies, lifetimes, and wave functions in a single shot. According to our calculations, the Sevast'yanov-Zykov potential energy model of $\mathrm{SF}_{6}-\mathrm{N}_{2}$ [20] supports, in the absence of a centrifugal term, 11 bound states, whose energy levels read (in ascending order) $-166.787,-125.426,-91.278,-63.746,-42.211,-26.024$, $-14.505,-6.934,-2.550,-0.537$, and $-0.0114 \mathrm{~cm}^{-1}$. Resonances with asymptotic wave function amplitude smaller than $1 / 20$ of the wave function's absolute maximum were considered long-lived. Although their presence was scarce, they started appearing at $J=14$ and persisted up to rotational levels as high as $J \approx 100$. The contribution of the bound and predissociating $\mathrm{SF}_{6}-\mathrm{N}_{2}$ dimers was found to be equal to $30 \%$ for the former and $10 \%$ for the latter relative to the total integrated cross section. To the best of our knowledge, such a great contribution due to bound or quasibound dimers has never been reported before.

A triangular function of $1.5 \mathrm{~cm}^{-1}$ width at half-maximum was used to imitate the apparatus function used in the experiment, which is the width expected at wavenumbers about as far away from the laser frequency as the position of the DRS band. In addition, a bell-shaped function of $10 \mathrm{~cm}^{-1}$ width was considered to account for the nearcomplete coalescence of the structure in which pressure effects have resulted at the low-frequency region of the spectrum. This point deserves some more attention. In fact, in our experiment the buffer gas $\left(\mathrm{N}_{2}\right)$ had to be compressed to many tens of atmospheres (whatever the Raman frequency was) in order to compensate for the weak DRS signal. Although spectra that are strictly binary were recorded by pinpointing integrated signals scaling rigorously as $\rho_{a} \rho_{b}$, pressure effects induced by the environment of the pairs $a-b$ could not be removed, since to do so would require the use of truly low gas pressures. The range of variations in the working pressure conditions was too short to allow us to reveal any meaningful trend in the bandwidth (see our preceding paper [5]), but pressure effects were strong enough to destroy any structure in the spectrum that would otherwise be observed at low frequencies at the zero-density limit [23]. According to [24], the simple collisional model leads to a Lorentzian profile with a damping constant of about $0.04 \mathrm{~cm}^{-1}$ and, therefore, a full width at half-maximum of approximately $0.1 \mathrm{~cm}^{-1}$ (see [25]). Given that total gas pressures of order 100 atm were used in our experiment, a full width of about $10 \mathrm{~cm}^{-1}$ at half-maximum seems like a realistic value for the bell-shaped function we used to convolve the full theoretical rototranslational spectrum.

Particularly gratifying was the fact that, once all aforementioned effects were included, the computed spectrum was found to exhibit a shape overall similar to the one found experimentally, with intensities lying entirely within the area of uncertainty. In Fig. 2 this area is indicated in gray. Theoretical spectra are shown in Fig. 3 (solid line), where the continuum-continuum contributions due to free pairs

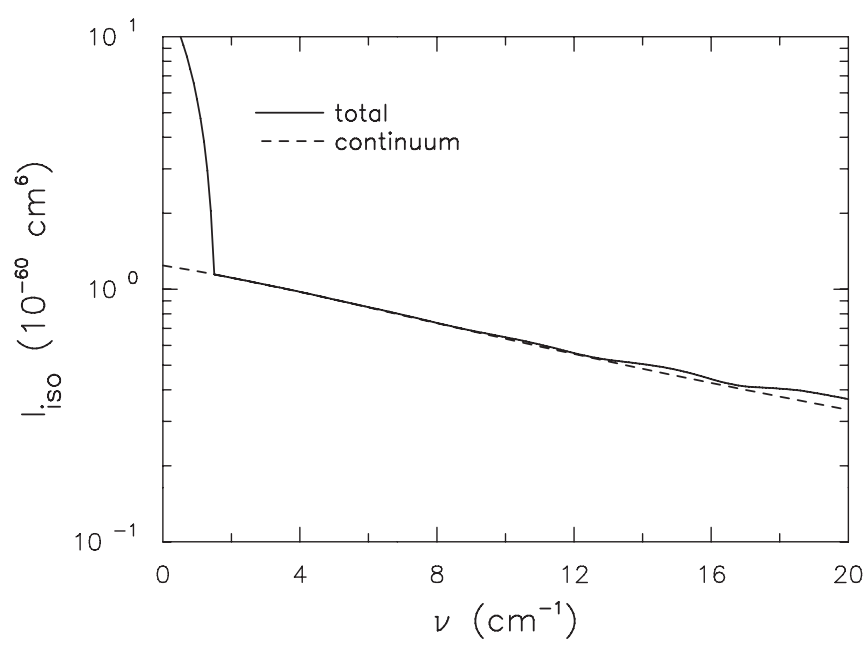

FIG. 3. Absolute isotropic spectrum $\left(\mathrm{cm}^{6}\right)$ computed with quantum mechanics as a function of frequency shift $\left(\mathrm{cm}^{-1}\right)$. A triangular apparatus function with full width at half maximum $1.5 \mathrm{~cm}^{-1}$ has been used but no function has yet been considered to account for the effects of pressure. Contributions by free pairs alone (dashed line); all contributions by free pairs and by bound and predissociating dimers (solid line). As entry parameter, the experimental value $C_{6}=247$ a.u. was used. 


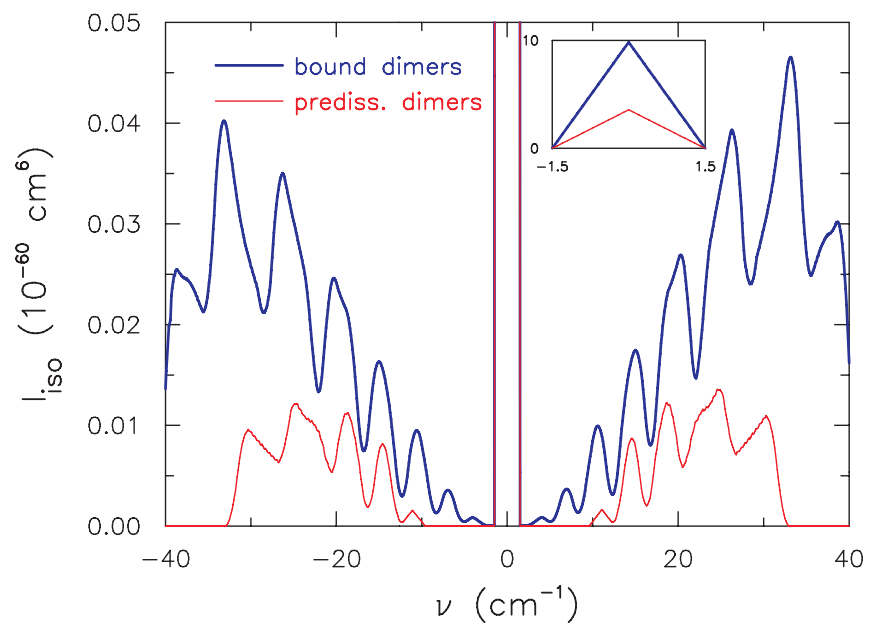

(a)

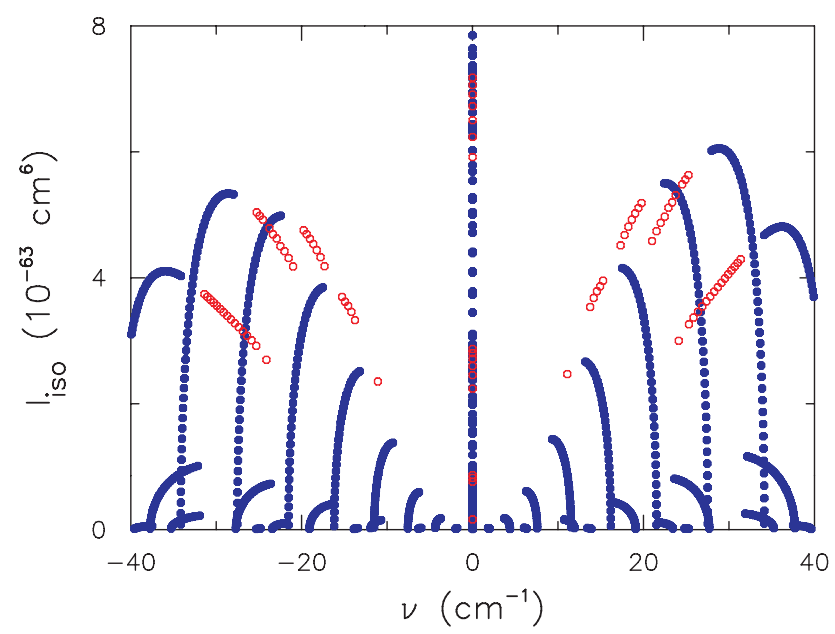

(b)

FIG. 4. (Color online) Absolute isotropic spectrum $\left(\mathrm{cm}^{6}\right)$ by bound and predissociating dimers, computed with quantum mechanics in the range $-40-40 \mathrm{~cm}^{-1}$ as a function of frequency shift $\left(\mathrm{cm}^{-1}\right)$. (a) A triangular apparatus function with full width at half-maximum of $1.5 \mathrm{~cm}^{-1}$ was considered (lines). (b) No apparatus function was considered (symbols). Bound dimers are shown in the respective panels by a blue (dark gray) line and by blue (filled) circles; predissociating dimers are shown by a red (light gray) line and by red (open) circles. The detailed balance principle manifests itself by the slight asymmetry seen between the Stokes side and the anti-Stokes side. The sharp peak around $v=0$ is shown separately (inset). Bound and predissociating dimers are responsible for more than one-third of the DRS intensity and their contribution comes predominantly from this peak.

(dashed line) have been superposed to the discrete contributions due to the bound and predissociating $\mathrm{SF}_{6} \mathrm{~N}_{2}$ dimers. Figure 4 separately illustrates, in the range $-40-40 \mathrm{~cm}^{-1}$, the rich bound and quasibound dimer structure with [Fig. 4(a)] and without [Fig. 4(b)] slit function. The slight asymmetry seen on this spectrum shows the expected effect of the detailed balance principle, which stems automatically from the computation.

According to our calculations, the large contribution of the bound and predissociating dimers, which as mentioned above were responsible for more than one-third of the total integrated intensity, comes predominantly from the sharp peak at $v=0$. However, what we see in reality as a result of pressure is a redistribution of this intensity over the unstructured continuum, which as illustrated in Fig. 2 makes intensities increase by almost a factor of 2 in the rather restricted frequency range covered by the experiment. Note that, if pressure effects were to be modeled explicitly, some pressure-narrowing effect unaccounted for in this paper would occur at the working conditions of gas density and pressure, and some additional intensity would move from the wings of the band to its center. The extent to which pressure narrowing affects the DRS bandshape remains to be seen [26].

It also remains to be seen to what extent relaxation of the point-polarizable molecule model affects the main characteristics of the isotropic spectrum. In this respect, it is worthwhile to note that, within a more realistic model going beyond the isotropic molecule approximation, the rotation of a

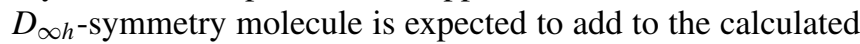
intensity. However, within the narrow spectral range, $|\nu| \lesssim$ $20 \mathrm{~cm}^{-1}$, where the isotropic signal was discernible against the noise background, only a minor part of the neglected effect is expected to be noticeable; for $\mathrm{N}_{2}$, the $M, O, S$, and $U$ rotational branches lie entirely outside that range. The issue raised above will be fully investigated, for both isotropic and anisotropic DRS, and is the subject of a forthcoming study [27].

\section{CONCLUSION}

In this paper we reported the weak isotropic collisioninduced Raman scattering component of the simultaneous vibrational transition $\mathrm{SF}_{6}\left(v_{1}\right)+\mathrm{N}_{2}\left(v_{1}\right)$ at room temperature. In spite of the noisy environment in which isotropic intensities were embedded, extraction of a meaningful line shape from the sparse data was possible. To this end, the property of the near-constant depolarization ratio $(0.83 \pm 0.02)$, which we observed over the narrow frequency range 3080-3120 $\mathrm{cm}^{-1}$, was used as a constraint. Agreement with predictions for integrated spectra was found on the basis of the point-polarizable molecule interaction model, provided that the DID and backinduction polarization mechanisms were supplemented with polarization dispersion and $\mathrm{DIQ}^{(2)}$ mechanisms, which for polarizability trace are of comparable significance to DID. The discrepancy from the experiment should mainly be attributed to the shortcomings of the point-polarizable molecule model, which in the case of the weak isotropic spectrum are expected to be more noticeable than in the case of the much stronger anisotropic spectrum. It might also be attributed to the $\mathrm{SF}_{6}-\mathrm{N}_{2}$ potential energy model, whose accuracy is still believed to be insufficient [28]. This discrepancy also showed strong dependence on certain employed parameters, especially on the rather sensitive Hamaker constant, with which intensity should scale quadratically. Quantum mechanics was used for frequency-resolved spectra, in agreement with the experiment. Finally, note that Unsöld-like approximations, such as the one made in passing from Eq. (5) to Eq. (7), are known [7] to underestimate dispersion effects in $\Delta \alpha_{000}$ by $\sim 20 \%$ or less. This suggests that Eq. (7) (and thus expressions resulting 
therefrom) provides a useful first approximation for dispersion effects but still would allow for an increase of up to $\sim 5 \%$ of the total isotropic intensity. Bound and predissociating dimers were found to contribute almost as much as the unstructured continuum, which at room temperature is, to the best of our knowledge, an unprecedented feature in the area of collision-induced spectroscopy. Through this paper, the issue of how to treat isotropic DRS comes within the scope of the general treatments of the reputed weak isotropic CIS spectra by gases and mixtures, which have repeatedly been the subject of attention by our group [2]. The results demonstrate that extremely unlikely events such as the ones reported here can indeed be seen by a Raman scattering experiment if given sufficient time to record the signal.

\section{ACKNOWLEDGMENTS}

Fruitful discussions with Dr. Alexander P. Kouzov, Dr. Tadeusz Bancewicz, and Dr. Florent Rachet are gratefully acknowledged.

\section{APPENDIX}

This appendix, addressed to wide audiences, is intended to give a brief and sharp demonstration of the DID expansion of the incremental trace $\Delta \alpha$ for an unlike atomic pair. To this end, electric fields are treated rather than the formalism of irreducible spherical tensors, which though powerful are friendly only to specialists.

Either of the two atoms $i(=a, b)$ is subject to external field $\overrightarrow{\mathcal{E}}$ and to the field $\vec{E}_{i j}$ generated by the dipole moment of the neighboring atom $j(=b, a)$. The dipole moment in each atom reads $\vec{\mu}_{i}=\alpha_{i} \vec{E}_{i}=\alpha_{i}\left(\overrightarrow{\mathcal{E}}+\vec{E}_{i j}\right)$. The sum $\vec{\mu}=$ $\vec{\mu}_{a}+\vec{\mu}_{b}=\vec{\mu}_{\text {ext }}+\vec{\mu}_{\text {int }}$ is identified as the dipole moment of the pair $a-b$, where $\vec{\mu}_{\text {ext }}=\left(\alpha_{a}+\alpha_{b}\right) \overrightarrow{\mathcal{E}}$ is the dipole moment associated with the external field and $\vec{\mu}_{\text {int }}=a_{a} \vec{E}_{a b}+\alpha_{b} \vec{E}_{b a}$ is the dipole moment owing to atom-atom interactions. Classical electromagnetism says that the field $\vec{E}_{i j}$, which is induced by atom $j$ at the position of atom $i$, reads

$$
\vec{E}_{i j}=\frac{3 \hat{n}_{j i}\left(\vec{\mu}_{j} \cdot \hat{n}_{j i}\right)-\vec{\mu}_{j}}{R^{3}},
$$

where $\hat{n}_{j i}$ designates the unit vector along the interatomic axis. By taking the two orthogonal orientations of the interatomic vector parallel $\left(\hat{n}_{j i} \| \vec{\mu}_{j}\right)$ and perpendicular $\left(\hat{n}_{j i} \perp \vec{\mu}_{j}\right)$ to the dipole moment $\vec{\mu}_{j}$, respectively, the latter formula can be written as two independent expressions:

$$
\begin{gathered}
\vec{E}_{i j \|}=2 \vec{\mu}_{j \|} R^{-3}=2 \alpha_{j}\left(\overrightarrow{\mathcal{E}}+\vec{E}_{j i \|}\right) R^{-3}, \\
\vec{E}_{i j \perp}=-\vec{\mu}_{j \perp} R^{-3}=-\alpha_{j}\left(\overrightarrow{\mathcal{E}}+\vec{E}_{j i \perp}\right) R^{-3} .
\end{gathered}
$$

By interchanging indices $i$ and $j$, one likewise has

$$
\begin{gathered}
\vec{E}_{j i \|}=2 \vec{\mu}_{i \|} R^{-3}=2 \alpha_{i}\left(\overrightarrow{\mathcal{E}}+\vec{E}_{i j \|}\right) R^{-3}, \\
\vec{E}_{j i \perp}=-\vec{\mu}_{i \perp} R^{-3}=-\alpha_{i}\left(\overrightarrow{\mathcal{E}}+\vec{E}_{i j \perp}\right) R^{-3} .
\end{gathered}
$$

Solving for $\vec{E}_{i j \|}$ and $\vec{E}_{i j \perp}$, we deduce

$$
\begin{aligned}
& \vec{E}_{i j \|}=\frac{1+\frac{2 \alpha_{i}}{R^{3}}}{1-\frac{4 \alpha_{i} \alpha_{j}}{R^{6}}} \frac{2 \alpha_{j}}{R^{3}} \overrightarrow{\mathcal{E}}, \\
& \vec{E}_{i j \perp}=-\frac{1-\frac{\alpha_{i}}{R^{3}}}{1-\frac{\alpha_{i} \alpha_{j}}{R^{6}}} \frac{\alpha_{j}}{R^{3}} \overrightarrow{\mathcal{E}},
\end{aligned}
$$

and likewise for $\vec{E}_{j i \|}$ and $\vec{E}_{j i \perp}$ upon interchange of $i$ and $j$.

On account of the expression $\vec{\mu}_{\text {int }}=\alpha_{a} \vec{E}_{a b}+\alpha_{b} \vec{E}_{b a}$ for the interaction part of the dipole moment, and of the definitions $\vec{\mu}_{\text {int } \|}=\alpha_{\|} \overrightarrow{\mathcal{E}}$ and $\vec{\mu}_{\text {int } \perp}=\alpha_{\perp} \overrightarrow{\mathcal{E}}$ in terms of the orientations $\|$ and $\perp$ that we defined above, one obtains

$$
\begin{aligned}
\alpha_{\|} & =\frac{1+\frac{\alpha_{a}+\alpha_{b}}{R^{3}}}{1-\frac{4 \alpha_{a} \alpha_{b}}{R^{6}}} \frac{4 \alpha_{a} \alpha_{b}}{R^{3}}, \\
\alpha_{\perp} & =-\frac{2-\frac{\alpha_{a}+\alpha_{b}}{R^{3}}}{1-\frac{\alpha_{a} \alpha_{b}}{R^{6}}} \frac{\alpha_{a} \alpha_{b}}{R^{3}} .
\end{aligned}
$$

By expanding $\Delta \alpha=\frac{1}{3}\left(\alpha_{\|}+2 \alpha_{\perp}\right)$ in a Taylor series, the following approximate expression of the incremental trace $\Delta \alpha$ is obtained to the $R^{-9}$ DID order:

$$
\Delta \alpha=\frac{1}{3}\left(\alpha_{\|}+2 \alpha_{\perp}\right) \approx 2 \alpha_{a} \alpha_{b}\left(\alpha_{a}+\alpha_{b}\right) R^{-6}+4 \alpha_{a}^{2} \alpha_{b}^{2} R^{-9} .
$$

This expression coincides up to the factor $\sqrt{3}$, as it should, with the one obtained [29] with irreducible spherical tensor formalism for the amplitude of the $\Delta \alpha^{(r)}$ tensor invariant of rank $r=0$.
[1] L. Frommhold, Adv. Chem. Phys. 46, 1 (1981); A. Borysow and L. Frommhold, ibid. 75, 439 (1989); L. Frommhold, Collision-Induced Absorption in Gases (Cambridge University, Cambridge, England, 1993); Collision- and Interaction-Induced Spectroscopy, edited by G. C. Tabisz and M. N. Neuman (Kluwer, Dordrecht, 1995); L. M. Trafton, in Molecular Complexes in Earth's Planetary, Cometary, and Interstellar Atmospheres, edited by A. A. Vigasin and Z. Slanina (World Scientific, Singapore, 1998).

[2] O. Gaye, M. Chrysos, V. Teboul, and Y. Le Duff, Phys. Rev. A 55, 3484 (1997); F. Rachet, Y. Le Duff, C. Guillot-Noël, and M. Chrysos, ibid. 61, 062501 (2000); S. Dixneuf, M. Chrysos, and F. Rachet, ibid. 80, 022703 (2009); M. Chrysos, S. Dixneuf, and F. Rachet, ibid. 80, 054701 (2009).
[3] Y. Le Duff, Phys. Rev. Lett. 90, 193001 (2003); J. Chem. Phys. 119, 1893 (2003).

[4] M. Zoppi, L. Ulivi, M. Santoro, M. Moraldi, and F. Barocchi, Phys. Rev. A 53, R1935 (1996).

[5] I. A. Verzhbitskiy, M. Chrysos, F. Rachet, and A. P. Kouzov, Phys. Rev. A 81, 012702 (2010).

[6] The scattering plane is the plane containing the incident light beam and the line from the center of the scattering system to the observer.

[7] K. L. C. Hunt, Y. Q. Liang, and S. Sethuraman, J. Chem. Phys. 89, 7126 (1988).

[8] Note that according to Hunt's Eq. (19) [7], and upon use of the relationship $\gamma_{X X X X}=3 \gamma_{X X Y Y}$, the isotropic invariant for nitrogen reads $\gamma_{b}=\frac{1}{15}\left(3 \gamma_{Z Z Z Z}+12 \gamma_{X X Z Z}+8 \gamma_{X X X X}\right)$, which 
is identical to the one given by Maroulis for this molecule [17]. However, in the case of $\mathrm{SF}_{6}$, the respective expressions given by the two authors differ from each other, namely $\gamma_{a}=\frac{3}{10}\left(3 \gamma_{Z Z Z Z}+\gamma_{X X Z Z}\right)$ [7] and $\gamma_{a}=\frac{3}{5}\left(\gamma_{Z Z Z Z}+2 \gamma_{X X Z Z}\right)$ [16], but they both reduce to $\gamma_{a}=\gamma_{Z Z Z Z}$ in the atom limit $\left(\gamma_{X X Z Z}=\frac{\gamma_{Z Z Z Z}}{3}\right)$.

[9] D. C. Hanna, M. A. Yuratich, and D. Cotter, Nonlinear Optics of Free Atoms and Molecules (Springer, New York, 1979).

[10] U. Hohm, Chem. Phys. Lett. 211, 498 (1993).

[11] F. London, Z. Phys. Chem. Abt. B 11, 222 (1930).

[12] S. Botti, A. Castro, X. Andrade, A. Rubio, and M. A. L. Marques, Phys. Rev. B 78, 035333 (2008).

[13] S. M. El-Sheikh, G. C. Tabisz, and A. D. Buckingham, Chem. Phys. 247, 407 (1999).

[14] R. T. Pack, J. Phys. Chem. 86, 2794 (1982).

[15] J. M. St. Arnaud and T. K. Bose, J. Chem. Phys. 71, 4951 (1979).

[16] G. Maroulis, Chem. Phys. Lett. 312, 255 (1999).

[17] G. Maroulis, J. Chem. Phys. 118, 2673 (2003).

[18] D. A. Long and E. L. Thomas, Trans. Faraday Soc. 59, 1026 (1963).

[19] A. Kumar, G. R. G. Fairley, and W. J. Meath, J. Chem. Phys. 83, 70 (1985).

[20] R. M. Sevast'yanov and N. A. Zykov, J. Eng. Phys. Thermophys. 40, 87 (1981). For details, see Ref. [19] in our preceding paper [5].

[21] M. Chrysos, O. Gaye, and Y. Le Duff, J. Phys. B 29, 583 (1996).

[22] M. Chrysos, O. Gaye, and Y. Le Duff, J. Chem. Phys. 105, 31 (1996).
[23] This is to be contrasted to standard rototranslational or vibrational CIS experiments with heavy gases, such as our recent work on Kr-Xe [S. Dixneuf, M. Chrysos, and F. Rachet, J. Chem. Phys. 131, 074304 (2009)]. There, pressures as low as a few atmospheres had been used to record CIS in the low-frequency range and had permitted the observation of a pronounced rotovibrational structure by bound and predissociating $\mathrm{Kr}-\mathrm{Xe}$ dimers.

[24] A. Thorne, U. Litzén, and S. Johansson, Spectrophysics: Principles and Applications (Springer, Berlin, 1999), pp. 204-206.

[25] This value is also corroborated by experimental broadening parameters for many atmospheric constituents broadened by air, which are indeed of order $0.1 \mathrm{~cm}^{-1} / \mathrm{atm}$.

[26] Such a treatment could be envisioned only if a "numerical experiment" were carried out through molecular dynamics simulations to get a complete view of the motion of the particles and of the way $a-b$ pairs scatter light in the presence of the surrounding particles.

[27] I. A. Verzhbitskiy, M. Chrysos, and A. P. Kouzov, Phys. Rev. A (to be published).

[28] While anisotropic intermolecular potential models have been developed for $\mathrm{SF}_{6}-\mathrm{Rg}$ [see, for instance, S. M. El-Sheikh, G. C. Tabisz, and R. T. Pack, J. Chem. Phys. 92, 4234 (1990)], there is no anisotropic potential model available for $\mathrm{SF}_{6}-\mathrm{N}_{2}$, even though the anisotropy of the interaction energy in the latter system is expected to be much greater than it is for the former systems. The development of an elaborated $\mathrm{SF}_{6}-\mathrm{N}_{2}$ potential energy surface would be welcome.

[29] A. P. Kouzov, M. Chrysos, F. Rachet, and N. I. Egorova, Phys. Rev. A 74, 012723 (2006). 